
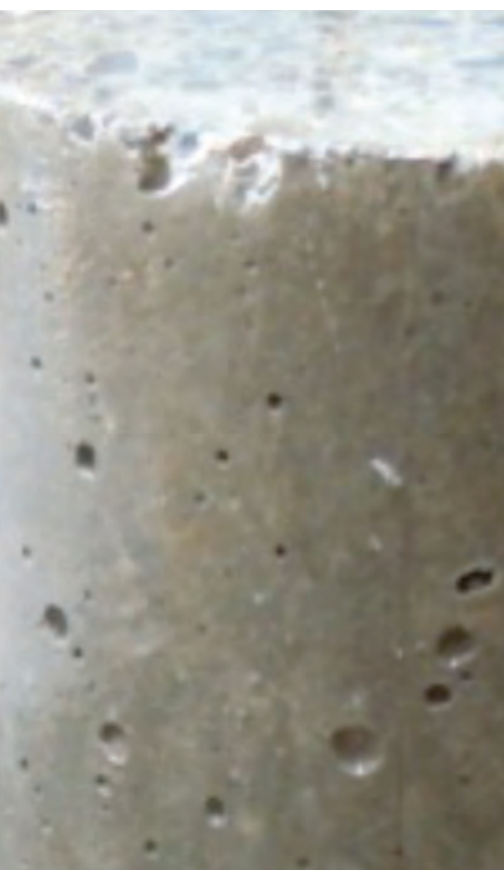

\title{
Recomendaciones para obtener resultados confiables de resistencia de cilindros de concreto
}

Fecha de recepción: 29 de agosto del 2011

Fecha de aceptación: 29 de setiembre del 2011

\section{Resumen}

A continuación se presenta una serie de recomendaciones para asegurar la confiabilidad de los resultados de resistencia de concreto muestreado en obra. Se describe cada uno de los pasos para lograr ese objetivo. Estos son: muestreo, moldeo, curado, protección y falla. Las recomendaciones brindadas se basan en un estudio profundo de las prácticas y normas así como en la experiencia adquirida en el proceso de falla de cilindros de concreto en el LanammeUCR. Este artículo está dirigido principalmente a los laboratorios de ensayo que ejecutan muestreos y pruebas de concreto pero también a los ingenieros y profesionales que trabajan en la construcción de obras civiles.

\section{Palabras clave:}

Concreto, resistencia a la compresión, muestreo de concreto, moldeo de especímenes de concreto, curado de concreto
Ing. Guillermo González Beltrán, PhD., LanammeUCR Ing. Ana Monge Sandí, MSc., Lanamme. UCR

\begin{abstract}
This paper is about a series of recommendations to assure the reliability of concrete strength results sampled in the field. Each of the steps to achieve this goal is described. Those are: sampling, molding, curing, protecting and testing. The provided recommendations are based on a deep study of the practices and standards as well as the experience acquired in the process of testing concrete cylinders at LanammeUCR. This paper is intended mainly for testing laboratories which execute sampling and testing of concrete but also for engineers and professionals that work in the construction of buildings.
\end{abstract}

\section{Keywords:}

Concrete, compressive strength, concrete sampling, molding concrete specimens, concrete curing

de la calidad de este material es de suma importancia para obtener obras civiles seguras.

Una de las maneras de realizar el control de la calidad de este material, es mediante la verificación de la resistencia 
a la compresión especificada por el diseñador de la obra. Por ello, han surgido laboratorios especializados que se encargan de realizar distintos ensayos para verificar precisamente este parámetro de calidad.

A pesar de que se trata de laboratorios especializados, se ha notado que el personal de los mismos no cuenta con la capacitación suficiente como para realizar los ensayos tal como son estipulados en las normas y más bien ejecutan los ensayos con prácticas aprendidas de manera deficiente y que pueden ocasionar resultados poco confiables al fallar especímenes de concreto muestreado y ensayado bajo esas condiciones.

Debido a esto, a continuación se presenta una descripción del proceso de obtención de muestras y se brindan recomendaciones y cuidados a tomar en cuenta al momento de realizar los ensayos para determinar la resistencia del concreto.

\section{Muestreo}

La primera etapa de la cadena de pasos para lograr un resultado exitoso en la falla de cilindros de concreto es el muestreo. La toma de muestras para conformar los especímenes debe hacerse de manera sistemática y siguiendo lineamientos establecidos de previo a cada muestreo específico. Las preguntas más comunes sobre el muestreo de concreto en obra son las siguientes:

¿Cuántas muestras deben tomarse durante el proyecto? ¿Dónde y en qué momento deben tomarse las muestras? ¿Cómo se deben tomar las muestras?

¿De qué tamaño deben ser las muestras?

Las respuestas a estas preguntas se pueden encontrar en los siguientes documentos: ACl-318 [1], ASTM C 172 [3] y ASTM C 94 [9]. A continuación se irán contestando estas preguntas, y a la vez se darán recomendaciones para diferentes situaciones que pueden ocurrir durante un muestreo.

\subsection{Cantidad y tamaño de muestras}

La cantidad de muestras de concreto mínimas que deben tomarse en una obra están establecidas en el capítulo 5 del ACl-318 [1]. Según este documento, se deben tomar muestras de cada clase de concreto al menos:

- Una vez al día

- Una vez por cada $110 \mathrm{~m}^{3}$

- Una vez por cada $460 \mathrm{~m}^{2}$ de losas o muros.
Cuando los requisitos anteriores producen 5 ensayos de resistencia o menos, para cada clase de concreto, debe muestrearse al menos 5 tandas al azar o todas las tandas cuando son menos de 5 .

Si el total de concreto de una clase que se va a colocar es menor que $38 \mathrm{~m}^{3}$ no es necesario muestrear para ensayos de resistencia si se demuestra con evidencia que la resistencia es adecuada.

Es recomendable que el ingeniero estructural de la obra establezca este plan, previo a la construcción, en las especificaciones del proyecto, así como las medidas a tomar en caso de resultados desfavorables. Los muestreos deben ser realizados por personal técnico calificado (certificado). Dependiendo de como se desarrolla la obra y los resultados que se van obteniendo, el ingeniero inspector puede incrementar el plan de muestreo establecido originalmente.

El tamaño de las muestras para ensayos de resistencia (compresión o flexión) debe ser de al menos $28 \mathrm{~L}$ por muestra combinada.

\section{$2.2 \quad$ Toma de muestras}

Las muestras de concreto se pueden tomar de varios puntos distintos dependiendo del propósito del muestreo. Por ejemplo, a una empresa productora de concreto le interesa tomar muestras de la mezcladora estacionaria para controlar la calidad de su producto y asegurarse que las proporciones y la calidad de los materiales de la mezcla es adecuada. Por otro lado, al comprador le interesa tomar la muestra justo antes de ser colocada en el sitio para que sea representativa del concreto colocado.

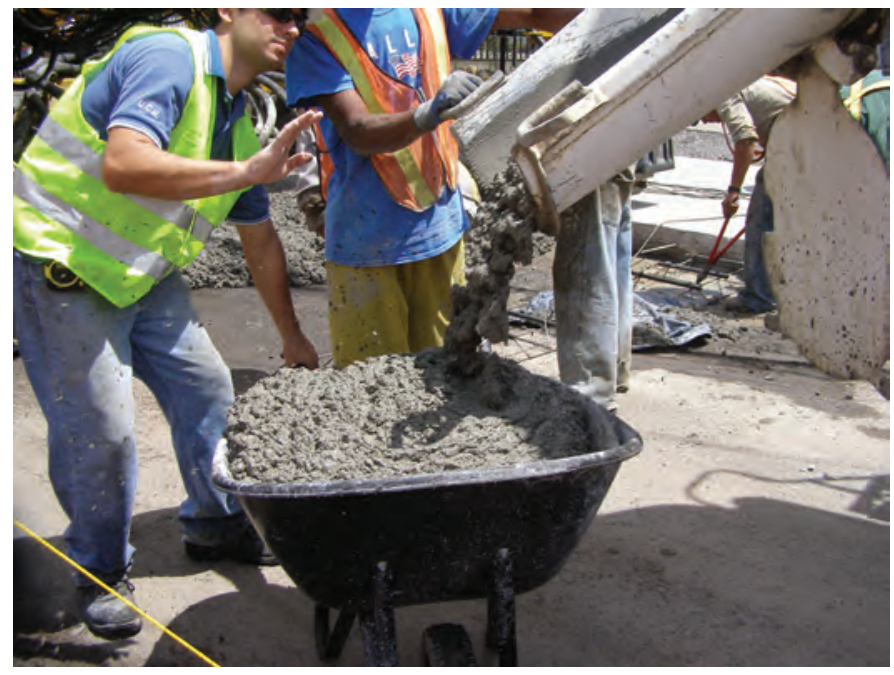

Figura 1. Muestreo en camión automezclador 
Es de suma importancia que el muestreo se realice una vez que se han hecho todos los ajustes a la mezcla, o sea, que se haya incluido todo el agua y aditivos.

Las muestras deben tomarse alrededor de la mitad de la descarga con el fin de obtener una muestra más representativa. Lo anterior se puede establecer estimando el tiempo total que dura la descarga. En el caso de las pavimentadoras, la muestra se toma después de haber descargado todo el concreto.

Las muestras se deben tomar en un recipiente limpio, impermeable, húmedo y con un tamaño adecuado para poder acomodar el concreto. Se recomienda utilizar carretillos de metal o plástico. Cuando se muestrea de un camión automezclador, se debe desviar la descarga directamente al recipiente y no se debe interrumpir para agilizar o retrasar la velocidad, pues esto puede provocar segregación (ver Figura 1). Se deben tomar al menos dos porciones espaciadas en intervalos regulares, de manera que se forme una muestra combinada con el tamaño adecuado para las pruebas de resistencia (compresión o flexión) y de control de calidad (revenimiento, temperatura, contenido de aire). No deben transcurrir más de quince minutos desde que se toma la primera porción hasta la última.

Una vez que se tiene la muestra compuesta, se debe trasladar al sitio donde se realizarán las pruebas, el cual debe ser una superficie nivelada y estar cerca del sitio donde se dará el curado inicial a los especímenes. Esto se debe definir antes de iniciar el muestreo. Se debe proteger la muestra contra contaminación y evaporación rápida (viento, sol). Antes de iniciar los ensayos, se debe remezclar con una pala para formar la muestra combinada y humedecer el equipo que va estar en contacto con el concreto.

Es importante verificar que se cuenta con agua potable en la zona donde se ejecutarán los ensayos. Los ensayos de resistencia deben iniciarse lo antes posible pero no después de 15 minutos de haber completado la muestra combinada. Se recomienda una vez que se tiene la muestra compuesta, iniciar con revenimiento y temperatura, luego seguir con contenido de aire y finalmente continuar con el moldeo de especímenes.

\section{Moldeo}

Otra etapa importante en la obtención de resultados confiables de resistencia de concreto es la elaboración de especímenes para ser fallados.
Para elaborar los especímenes cilíndricos de concreto, se deben cumplir estándares establecidos para estos propósitos tales como los estipulados en la norma ASTM C 31 [4], en la cual se indica el procedimiento a seguir para elaborar especímenes en el campo, es decir en el sitio de la obra, representativos del concreto muestreado.

Por lo tanto, es importante que el personal que se encargue de la elaboración de los especímenes reciba un buen entrenamiento en estos procedimientos y además, que conozcan las razones generales de por qué se deben elaborar los especímenes de manera estándar para garantizar los resultados de resistencia confiables. Para ello, existen programas internacionales tales como la certificación de técnicos por medio del American Concrete Institute (ACl) donde se imparten cursos en diferentes países, con el fin de entrenar a los técnicos en ensayos de campo y de laboratorio de tal forma que se garantice la representatividad del concreto colocado en la obra.

\subsection{Moldes}

Lo primero que se establece en la norma ASTM C31[4] es que los moldes que se utilicen para la elaboración de los especímenes deben ser rígidos, no absorbentes, que cumplan con el requisito de estanqueidad que establece la norma ASTM C 470 [6], entre otros. También, deben ser capaces de soportar las condiciones de trabajo durante los muestreos realizados en el campo. Los moldes metálicos han resultado ser adecuados para este tipo de trabajos.

Un aspecto importante que no se debe dejar de verificar en los moldes es la perpendicularidad. Esta debe cumplir el requisito de $0.5^{\circ}$ con respecto al eje del molde. Este es un requisito que también se debe verificar en los especímenes de concreto con una escuadra calibrada, (ver Figura 9a).

\subsection{Consolidación}

Para el moldeo de los especímenes en campo es que deben realizarse colocando el concreto en el molde en capas de igual volumen, esto con el fin de eliminar la segregación de los materiales que componen el concreto y no generar problemas de distribuciones de esfuerzos no uniformes al momento de realizar la falla del espécimen.

La consolidación del concreto en cada capa debe realizarse de tal manera que se garantice que los vacíos entre partículas sean lo menos posibles para que no afecte negativamente la resistencia. Además, con una adecuada consolidación se logra aumentar la densidad del espécimen de concreto y esto ayuda a conseguir resistencias adecuadas al realizarse la falla de los cilindros. La consolidación se puede realizar 
por medio de apisonamientos con varilla o bien con vibradores.

Cuando se va a consolidar el espécimen por medio de apisonamientos con la varilla, para el caso de especímenes de $150 \times 300 \mathrm{~mm}$, se debe colocar el concreto en tres capas y para el caso de especímenes de 100×200 mm, en dos capas. Se procede a realizar la consolidación aplicando 25 golpes asegurándose que se está penetrando toda la capa, incluso se recomienda que la varilla penetre la capa inmediatamente anterior unos $25 \mathrm{~mm}$ aproximadamente. Finalmente al terminar cada apisonamiento de capa, se deben aplicar de 10 a 15 golpes con un mazo con el fin de terminar de cerrar los vacíos que pudo haber dejado el apisonamiento de la varilla y con ello aumentar la densidad.

Para el caso de consolidar el concreto con vibrador, el número de capas es de dos para cualquier tamaño de molde, pero las inserciones son una para el molde de 100×200 mm, y dos para el molde de $150 \times 300 \mathrm{~mm}$. Igualmente, se debe golpear con el mazo de 10 a 15 golpes.

Además, cuando se realice la consolidación con la varilla, se debe tomar en cuenta no propinar los golpes de una manera ligera y débil, pues entonces no se logrará la consolidación apropiada del concreto; pero tampoco se debe hacer de una manera muy vigorosa pues lo que sucede es que se genera segregación de los agregados gruesos y se obtendrían valores menores que los especímenes consolidados adecuadamente.

Igualmente, al momento de realizar las inserciones del vibrador, se debe tener cuidado de no sobre-vibrar el concreto para no generar segregación. Esto se puede evitar conociendo el tiempo que se debe permitir al vibrador estar inmerso en la capa de concreto.

Por último, al momento de propinar los golpes con el mazo, se debe cuidar de no hacerlo muy fuerte pues se puede perder concreto e incluso se pueden generar otros vacíos importantes en las capas consolidadas. $Y$ también debe cuidarse de no hacerlo en forma débil, pues no terminará de cerrar los vacíos dejados por la varilla o el vibrador al terminar la consolidación.

\subsection{Acabado}

Para terminar, es importante mencionar el acabado que deben tener los especímenes al final de su elaboración. Este debe proporcionar una capa lo suficientemente lisa y plana como para que al momento de la falla no se vayan a generar concentraciones de esfuerzos.
Si se siguen todos los pasos del procedimiento para elaborar especímenes de concreto, tomando en cuenta lo mencionado anteriormente, se puede garantizar que el moldeo no será el factor que afecte los resultados de la resistencia de los especímenes.

\section{Curado y protección}

La cura y protección de los especímenes moldeados tanto en su estado fresco como endurecido, son importantes para que no se vean afectados los resultados de resistencia obtenidos a partir de la falla de los mismos. Cabe destacar que en la etapa de curado los especímenes de concreto desarrollan la resistencia con el tiempo, y este desarrollo de resistencia depende mucho del proceso de hidratación del cemento dentro de la masa de concreto. Si se cuenta con un curado apropiado, el cemento puede hidratarse continuamente y desarrollar la reacción química que genera la resistencia con el tiempo. Si el curado es deficiente, el cemento no se hidrata adecuadamente y la resistencia de diseño es probable que no se llegue a alcanzar.

Igual que con el moldeo, existen estándares establecidos para realizar protecciones y curados apropiados a los especímenes. Para el caso de los especímenes moldeados en campo, estos parámetros se especifican en la norma ASTM C31[4].

En esta norma, se indican las condiciones de humedad relativa y temperatura que se deben controlar para generar las condiciones ambientales apropiadas para que los especímenes moldeados de concreto, desarrollen la resistencia requerida para la aceptación de un concreto muestreado en obra.

Estas condiciones se deben tomar en cuenta tanto en la etapa del curado inicial como en la etapa del curado final.

\subsection{Curado inicial}

En cuanto al curado inicial la norma indica que se debe proteger el espécimen de pérdida de humedad en los moldes por un máximo de 48 horas, por medio de la colocación de bolsas plásticas cubriendo la superficie del espécimen, colocando tapas plásticas, o bien cuando las condiciones son muy adversas (altas temperaturas o climas muy secos), se puede proteger colocando arena húmeda o arpillería húmeda alrededor del molde.

Para esta etapa se recomienda utilizar un higrotermómetro junto a los especímenes de concreto recién moldeados, para monitorear la temperatura durante este período. 
El curado final es el que la norma clasifica de dos maneras: curado en campo o curado estándar. El curado en campo, se realiza para verificar cuándo retirar la formaleta, para determinar el momento en que se puede poner en uso la estructura, comparaciones con el curado estándar, entre otros.

El curado estándar es el que se realiza bajo las condiciones establecidas por la norma. En este caso, se debe mantener húmedo el espécimen en todo momento hasta que se realice la falla. Esta condición es la más sencilla de conseguir en el laboratorio, pues resulta más complejo establecer una relación entre la condición seca al aire y la resistencia. En este caso, la humedad relativa y la temperatura son las condiciones que se controlan.

Cuando se requiere aceptar la resistencia del concreto en la obra, los especímenes deben ser curados de manera estándar.

Para cumplir las condiciones del curado estándar, se recomienda que los especímenes permanezcan en un cuarto o cámara húmeda, o sumergidos en pilas, bajo las condiciones ambientales determinadas en la norma ASTM C $511[7]$.

\subsection{Condiciones ambientales}

En cuanto a las condiciones ambientales, la humedad relativa es un factor que influye directamente en el desarrollo de la resistencia. Se ha llegado a demostrar que para humedades relativas mayores de $60 \%$ no se presenta un movimiento de

Figura 2. Relación de la pérdida de agua del concreto y humedad relativa [10]

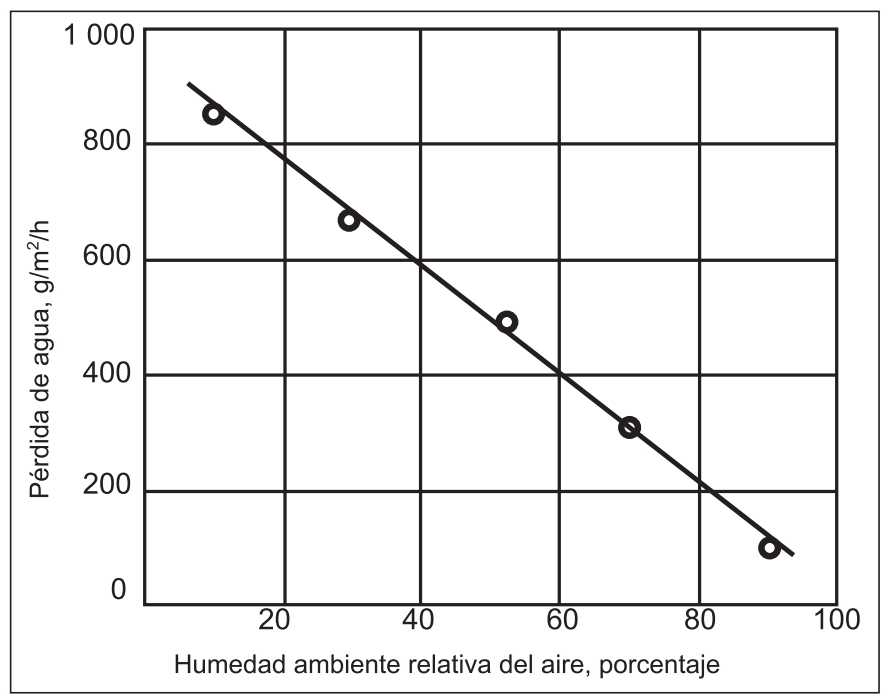

agua fácil entre el concreto y el aire del ambiente, evitando pérdidas importantes de agua en el concreto.

En la figura 2 se muestra cómo la pérdida de la cantidad de agua del concreto disminuye conforme aumenta la humedad relativa del ambiente, que se controla durante el proceso de curado de los especímenes. Evidentemente esta disminución en la pérdida de agua en el concreto contribuye a que se presente una adecuada y casi completa hidratación del cemento dentro de la masa de concreto, desarrollándose la resistencia con el tiempo de una manera apropiada.

La temperatura también es un factor que contribuye a un apropiado curado para el concreto. Se ha encontrado que a mayor temperatura ambiente, mayor es la pérdida de

Figura 3. Relación entre la pérdida de agua del concreto con la temperatura [10]

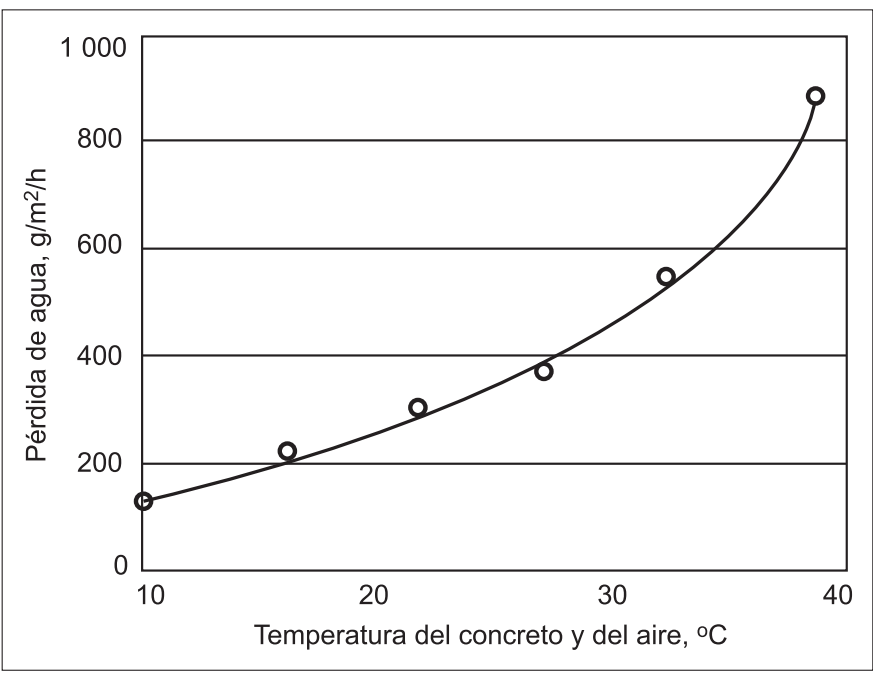

agua del concreto. Esto es perjudicial pues no se genera un ambiente apropiado para que el cemento continúe su proceso de hidratación en el tiempo. Esto puede observarse en la figura 3.

Incluso, la temperatura propia del concreto también afecta el desarrollo de su resistencia. Si el concreto desarrolla una alta temperatura por el proceso mismo de hidratación del cemento y no se cura de manera apropiada, el aumento de esta temperatura da cabida a menores desarrollos de resistencia. Este fenómeno se observa en la figura 4, donde la pérdida de agua que se puede presentar en el concreto puede ser más del doble, con el aumento de menos de $10^{\circ} \mathrm{C}$.

Otro factor que puede influir en que se presente un apropiado proceso de curado es el viento. Como se puede observar en la figura 5, entre mayor es la velocidad del viento, mayor es la pérdida de agua que se puede presentar 
Figura 4. Relación de la temperatura del concreto con la pérdida de agua [10]

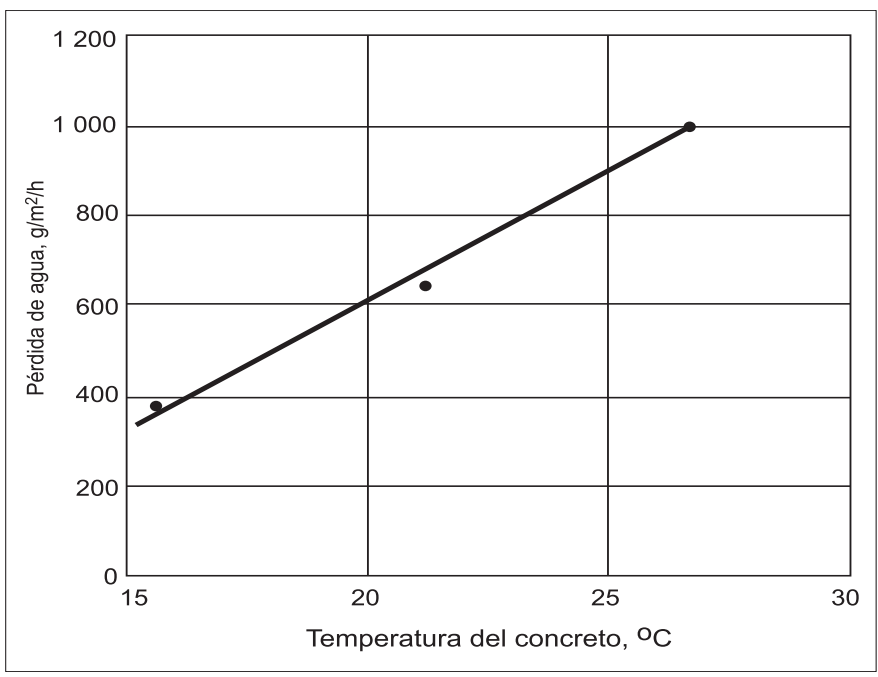

Figura 5. Relación entre la velocidad del viento y la pérdida de agua del concreto [10]

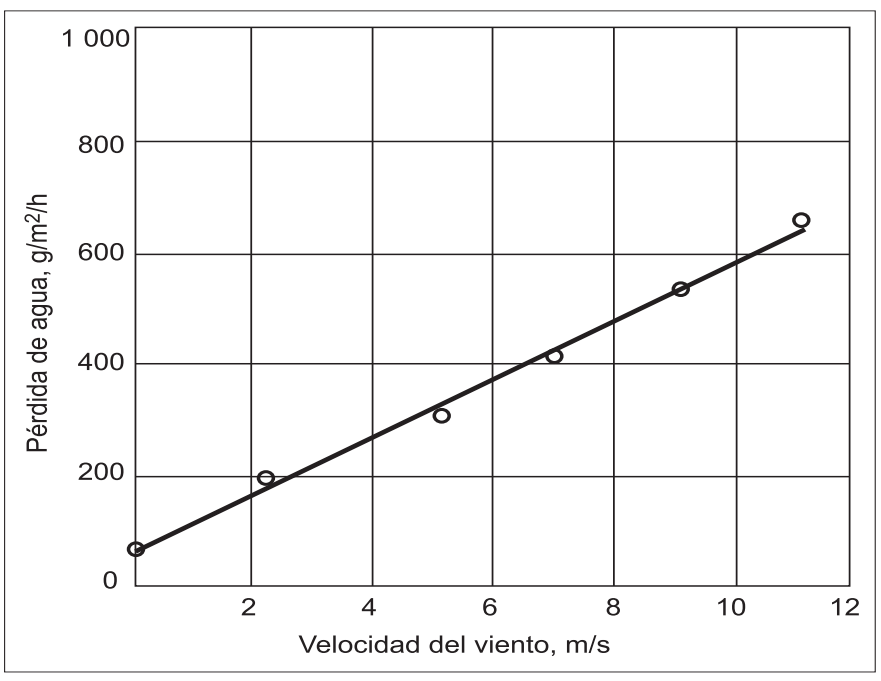

en el espécimen, dificultando el proceso apropiado de la hidratación del cemento dentro de la masa de concreto, y por lo tanto influyendo de manera negativa en el desarrollo de resistencia del concreto con el tiempo.

Por tal razón, la protección que se aplique a los especímenes de concreto para evitar la pérdida de agua es de suma importancia.

\subsection{Protección}

Una vez recién moldeados los especímenes, se debe tener cuidado de que se encuentren protegidos durante el curado inicial. Esta protección debe ser contra la exposición directa a los rayos solares, la evaporación, el viento y la lluvia. En caso de que el sitio en el que se realiza el moldeo y en que reciben el curado inicial cuenta con condiciones climáticas adversas, se recomienda acondicionar un sitio con humedad relativa y temperatura controlada.

Durante el transporte del sitio de muestreo al laboratorio, el cual debe realizarse no antes de 8 horas después del tiempo de fragua final, los especímenes se deben proteger adicionalmente de recibir golpes durante el viaje, ya sea con los mismos especímenes o bien con el vehículo que los transporta. Para evitar esta condición, se recomienda colocar los especímenes en moldes especiales acolchados, rodearlos con arena húmeda o telas húmedas. No hay que olvidar protegerlos también contra la pérdida de humedad.

\section{Falla}

El último paso para completar un resultado confiable de resistencia del concreto es la falla del espécimen. La norma ASTM C 39 [5] describe el procedimiento de falla para especímenes cilíndricos de concreto. Si se asume que los pasos anteriores (muestreo, moldeo y curado) se ejecutaron adecuadamente, el resultado de la falla de un espécimen cilíndrico de concreto puede verse afectado por varios factores tales como: tamaño, tamaño máximo del agregado, razón entre longitud y diámetro, velocidad de deformación, rigidez de los bloques de carga, ubicación de la carga, planicidad de las caras extremas y condición de humedad del espécimen.

\subsection{Tamaño del espécimen}

La resistencia del concreto se ve afectada por el efecto de tamaño, el cual es un fenómeno en el que se observa que entre menor es el tamaño del espécimen, mayor es su resistencia. La forma del espécimen también influye en el resultado de resistencia. Por ejemplo, un cilindro de concreto de $150 \times 300 \mathrm{~mm}$ alcanza una resistencia aproximada del $80 \%$ de la que se obtiene con un espécimen cúbico de $150 \mathrm{~mm}$ de lado, por el efecto de restricción de placas. En concretos de alta resistencia el factor de $80 \%$ aumenta.

Por las razones anteriores, la norma ASTM C 39 [5] define una forma (cilíndrica) y dos tamaños $(150 \times 300$ mm y 100×200 $\mathrm{mm}$ ) para el espécimen normalizado. La forma cilíndrica se ha preferido debido a una mayor uniformidad por un efecto menor de la restricción de placas, mejor distribución de los esfuerzos en planos horizontales y menor efecto de pared del agregado grueso en la resistencia.

La diferencia de resistencia entre los dos tamaños de cilindros no es significativa y su escogencia depende del tamaño máximo 
del agregado. Es recomendable utilizar el tamaño de 100×200 $\mathrm{mm}$ siempre que sea posible, pues tiene ventajas como facilidad de manipulación, menos propensos a daños, menor costo de moldes, utilización de una máquina de menor capacidad, menos concreto y menos espacio para almacenamiento y curado.

\subsection{Tamaño máximo del agregado}

El tamaño máximo del agregado puede afectar la resistencia debido a que se genera un efecto de tamaño que no es representativo del concreto. El espacio entre el agregado grueso y la pared es mayor que en el centro y se requiere mayor cantidad de mortero para rellenarlo. El efecto de pared es mayor cuando la razón entre superficie y volumen es mayor. Debido a lo anterior, el tamaño máximo del agregado se limita a $D / 3$, donde $D$ es el diámetro del cilindro.

\subsection{Razón entre longitud y diámetro (L/D)}

Los especímenes que se utilizan para ensayos de compresión deben tener una razón de esbeltez baja para evitar los efectos de estabilidad (pandeo). Entre mayor es la razón de esbeltez, menor es la resistencia del espécimen. En la figura

Figura 6. Relación entre la resistencia relativa y la razón L/D

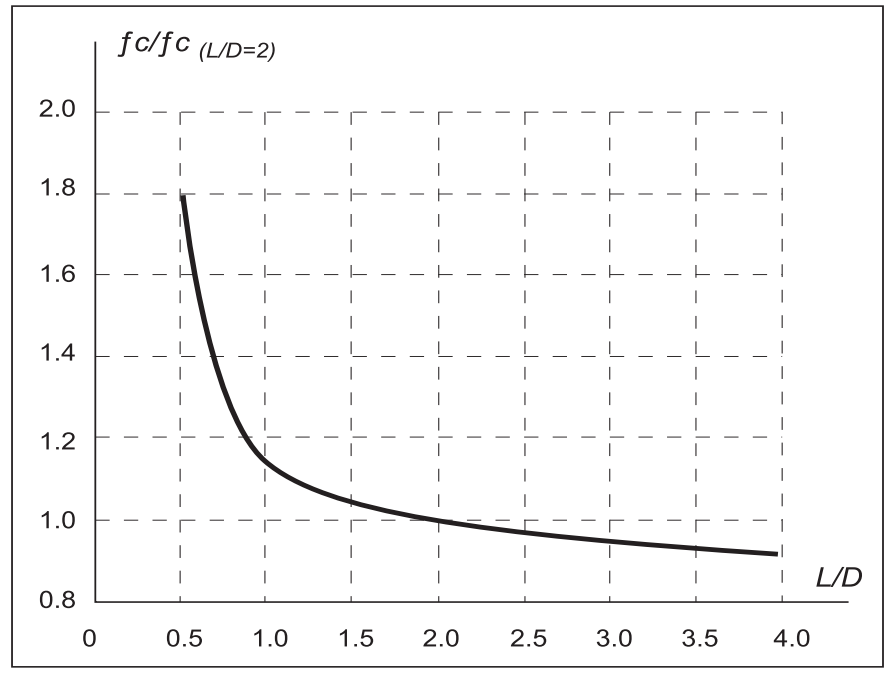

6 se muestra la relación entre la resistencia en compresión relativa de un espécimen cilíndrico de concreto y la razón L/D (que es una medida indirecta de la razón de esbeltez). Se puede observar en esta figura que conforme aumenta la relación L/D, disminuye la resistencia en compresión.

El valor normalizado para la relación L/D que se define en la norma ASTM C 39 [5] es de 2.0. Este valor es conveniente, pues como se puede notar en la figura 6 , la curva decrece rápidamente en resistencia conforme aumenta la relación
L/D hasta un valor aproximado de 1.50, a partir del cual, la resistencia decrece lentamente. Lo anterior se debe a que conforme disminuye la razón $L / D$, el efecto de la restricción de placas en los extremos es mayor y por lo tanto aumenta la resistencia. Se ha observado que para concretos de alta resistencia este efecto es menor.

Figura 7. Relación entre la resistencia relativa y la razón f ${ }_{c / t}$

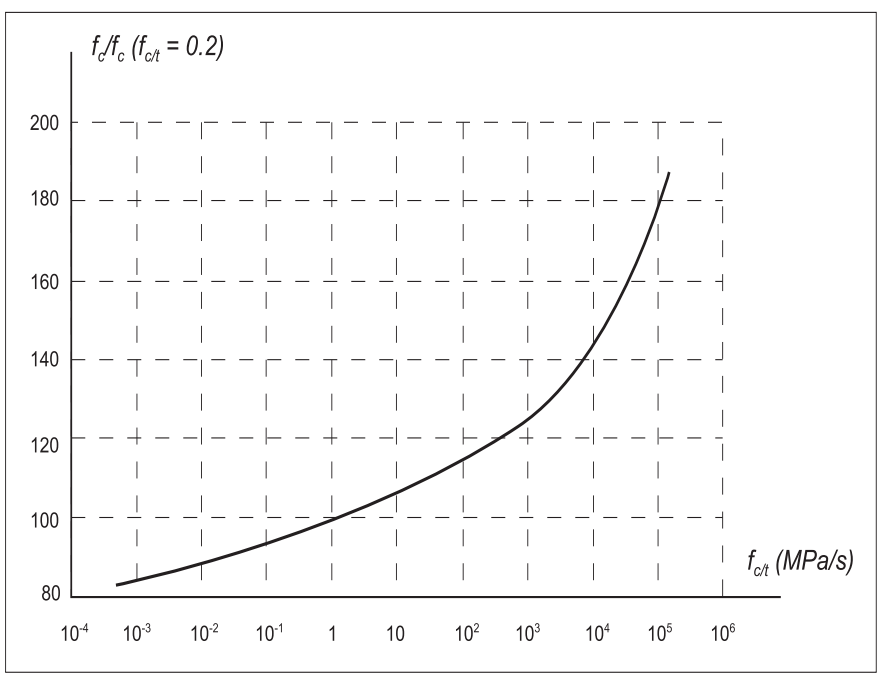

\subsection{Velocidad de deformación}

El concreto es un material viscoelástico, lo cual quiere decir que su deformación aumenta con el tiempo para un esfuerzo constante. Este fenómeno se conoce como flujo plástico y provoca que la resistencia aumente conforme aumenta la velocidad de deformación. La figura 7 muestra la variación de la resistencia del concreto en compresión con la velocidad de esfuerzo.

La norma ASTM C 39 [5] establece la velocidad de deformación en términos de velocidad de esfuerzo, la cual debe mantenerse entre $0.2 \mathrm{MPa} / \mathrm{s}$ y $0.3 \mathrm{MPa} / \mathrm{s}$ en la segunda mitad de la fase de carga estimada. Por ejemplo, un espécimen de $150 \times 300 \mathrm{~mm}$ y $28 \mathrm{MPa}$ de resistencia estimada debe durar entre 47 y 70 segundos en la segunda fase de carga (a partir de $14 \mathrm{MPa}$ ). En la primera fase de carga, la velocidad no está establecida pero hay que asegurarse que la carga sea continua y sin golpes.

En máquinas con servo control, se programa la velocidad en términos de esfuerzo o de carga. En este caso, se puede mantener la velocidad constante durante todo el proceso. Para el espécimen del ejemplo anterior, la velocidad de carga estaría entre $3.53 \mathrm{kN} / \mathrm{s}$ y $5.30 \mathrm{kN} / \mathrm{s}$. Sin embargo, se recomienda programar la velocidad en términos de esfuerzo, 
pues en este caso se puede utilizar para cualquier tamaño de espécimen.

En máquinas con control manual, existe la posibilidad de introducir el diámetro del espécimen y observar en la pantalla del indicador la velocidad en términos de esfuerzo ( $\mathrm{MPa} / \mathrm{s})$. En este caso, la primera mitad de la fase de carga (de $0 \mathrm{MPa}$ a $14 \mathrm{MPa}$ ) sirve para ajustar la velocidad manualmente y aproximarla a los valores indicados en el rango.

Para las máquinas que solo tienen indicador analógico de carga, es necesario fallar un cilindro de prueba para ajustar la velocidad al tiempo requerido. Para el espécimen del ejemplo, se debería durar entre 47 y 70 segundos entre la carga de $247 \mathrm{kN}$ (mitad de la carga estimada) y 495 kN (carga de falla estimada). Para este tipo de máquinas, cuando la carga de falla estimada difiere significativamente de la carga estimada, se debe calcular de nuevo el tiempo con la carga obtenida.

\subsection{Rigidez de los bloques de carga}

Para evitar concentraciones de esfuerzos, la norma ASTM C 39 [5] indica que se debe colocar una cabeza con asiento esférico entre la parte superior del espécimen y el bloque de carga superior. Esto implica que se debe definir un espesor del bloque de carga para evitar deformaciones excesivas en el mismo.

La norma ASTM C 39 [5] define el espesor mínimo que deben tener los bloques de carga. Esto se debe a que si las placas no son lo suficientemente rígidas con respecto al espécimen, pueden deformarse y producir concentraciones de esfuerzos en las caras extremas del concreto, provocando un esfuerzo de falla menor al que se obtiene con placas rígidas.

También es importante asegurar la planicidad de los bloques de carga, la cual se indica en la norma $(0.05 \mathrm{~mm}$ para placas en uso y $0.025 \mathrm{~mm}$ para placas nuevas). La planicidad de los bloques de carga se puede comprobar con equipos de precisión. Se recomienda revisar la planicidad de las placas en un taller de precisión sino se cuenta con el equipo adecuado. Si las placas no son planas, también provocan concentraciones de esfuerzos en los extremos del cilindro, provocando una falla prematura.

\subsection{Ubicación de la carga}

En cualquier ensayo de tracción o compresión, la línea de carga debe coincidir con el centroide de la sección del espécimen para asegurar una carga concéntrica. Lo anterior evita que se introduzca excentricidad en la carga y que se provoque una falla no deseada debido a la flexión inducida por la excentricidad.

Por lo tanto, el cilindro de concreto se debe alinear con el eje de carga de la máquina de ensayos. Para lograr este

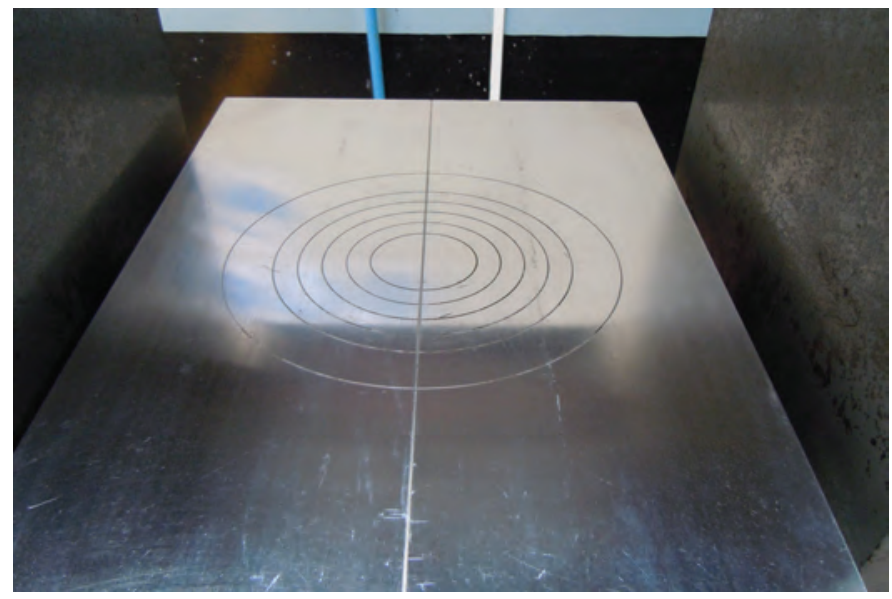

Figura 8. Círculos concéntricos en bloques de carga de máquina de falla

alineamiento, la mayoría de los bloques de carga poseen círculos concéntricos de diferentes diámetros en la superficie (ver figura 8). La norma ASTM C 39 [5] establece los requisitos para las dimensiones de las marcas de los círculos (profundidad y ancho).

\subsection{Planicidad de las caras extremas del espécimen}

En un ensayo de compresión, se requiere producir un esfuerzo constante en la sección transversal del espécimen. Para lograrlo, es indispensable que tanto las caras de los bloques de carga y soporte, y las caras del espécimen sean planas y paralelas. Si las caras no son lo suficientemente planas, se genera una concentración de esfuerzos en la superficie de las caras extremas y se obtiene un esfuerzo de falla inferior.

La norma ASTM C 39 [5] establece que la planicidad de las caras extremas del espécimen debe ser de $0.05 \mathrm{~mm}$ en $150 \mathrm{~mm}$, igual que la exigida para los bloques de carga y soporte en uso. Esta planicidad se puede comprobar con una regla y galga calibradas. Se coloca la regla calibrada sobre la superficie que está siendo comprobada y se intenta introducir la galga calibrada de $0.05 \mathrm{~mm}$. Si la galga no se puede introducir, quiere decir que la superficie cumple con la planicidad requerida, por otro lado, si se logra introducir la galga, quiere decir que la superficie no cumple con la 


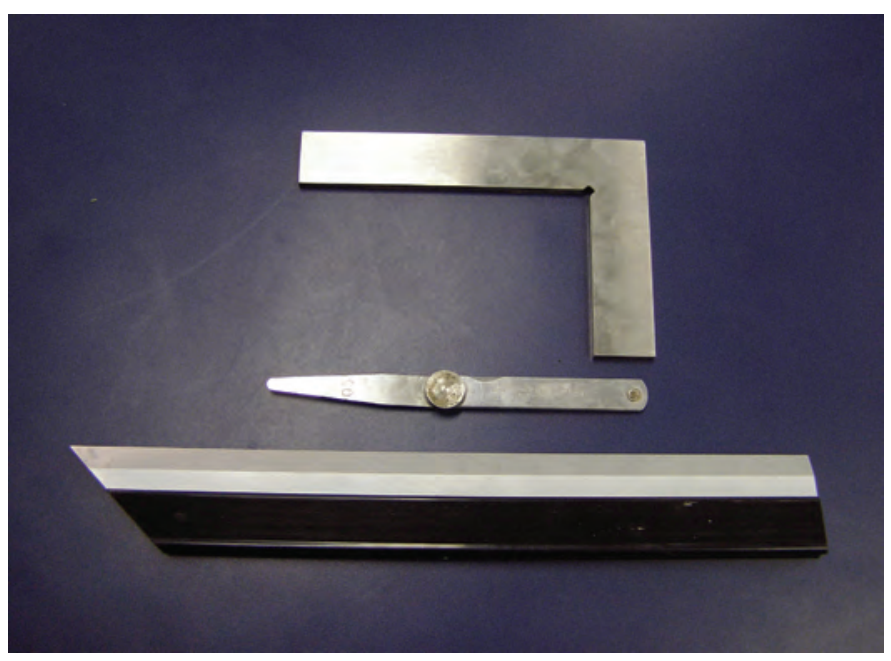

(a) Equipo para determinar la planicidad y perpendicularidad. De arriba hacia abajo:

1. Escuadra, 2. Galgas calibradas y 3. Regla calibrada

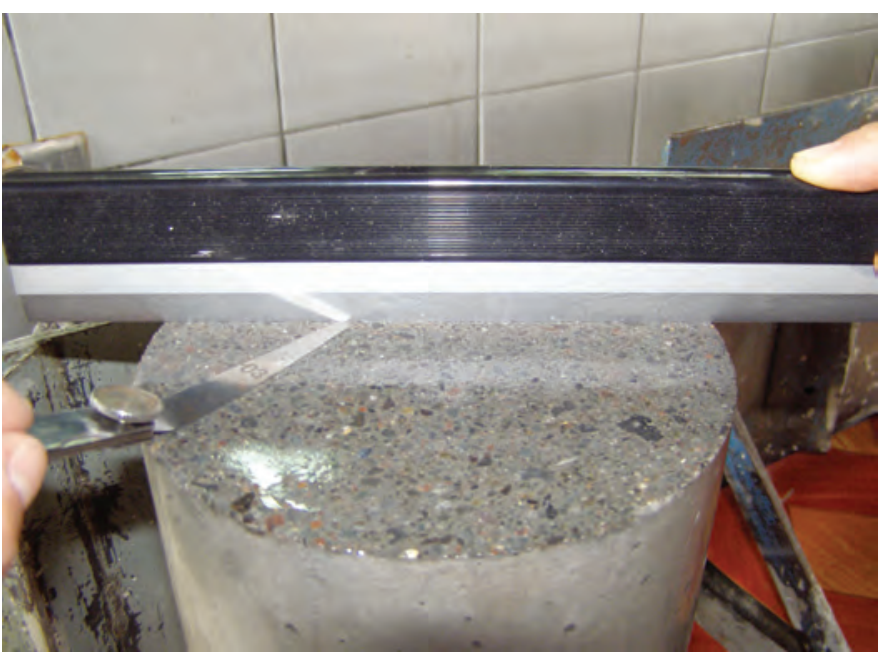

(b) Determinación de planicidad

Figura 9. Equipo y determinación de planicidad

planicidad requerida. En la figura 9 se muestra el equipo (a) y un ejemplo de su utilización (b).

En caso de que los especímenes no cumplan con la planicidad establecida, la norma permite que se coronen (ASTM C 617 [8]) o se fallen con coronas no adheridas tales como almohadillas de neopreno (ASTM C 1231 [2]). El coronamiento de los especímenes está fuera del alcance de este artículo.

\subsection{Condición de humedad del espécimen}

La norma ASTM C 39 [5] especifica que una vez que los especímenes son retirados de la cámara húmeda, deben fallarse lo antes posible y en condición húmeda. Lo anterior se debe a que si el espécimen pierde humedad su resistencia disminuye y es más sencillo mantener el espécimen húmedo que definir algún grado de sequedad.

También se ha observado que un espécimen seco al horno resiste más que un espécimen húmedo, y que si el espécimen seco al horno se humedece hasta obtener la condición de humedad inicial, se obtiene la misma resistencia que se hubiera obtenido si se fallara al sacarlo de la cámara húmeda.

Se recomienda que cuando se fallen muchos cilindros a la vez, se mantengan bien húmedos con un rociador de agua y que en el momento de la falla se asegure que el espécimen se encuentra totalmente húmedo (ver figura 10).

\section{Recomendaciones}

Después de estudiados los factores que pueden afectar la resistencia de cilindros de concreto y por lo tanto, la confiabilidad de los resultados, se enumeran a continuación las recomendaciones:

-Es necesario definir el plan de muestreo de concreto para la obra en estudio. Este plan debe incluir el número de ensayos de control de calidad y resistencia, según las cantidades de concreto colocado en obra, así como las medidas a tomar en caso de resultados desfavorables.

-Es importante que el personal del laboratorio que realiza los muestreos y los ensayos, se encuentre certificado por un ente reconocido nacional o internacionalmente para la formación de técnicos en ensayos de concreto, por ejemplo el ACl.

-Asegurarse que al momento de trasladarse al sitio de muestreo, se cuente con todo el equipo y herramientas necesarios para realizar los ensayos requeridos en el plan de muestreo.

-Tomar muestras lo más representativas posibles del concreto colado en obra, así como tomar las medidas preventivas durante la realización de los ensayos de control de calidad, así como de los especímenes moldeados en el campo.

- Tomar en cuenta que al momento de realizar el moldeo de los especímenes, se debe consolidar el concreto de 
una manera apropiada tal que no produzca segregación o vacíos que afecten la resistencia.

-Durante el curado inicial y final, no se debe olvidar tomar registros de las condiciones ambientales, para verificar que estas se encuentran de acuerdo con lo establecido.

Adicionalmente, se debe tener especial cuidado en la protección de los especímenes sobretodo en la etapa de curado para evitar pérdida de agua en la masa de concreto y durante el transporte impedir que se dañen.

En el proceso de falla, los aspectos primordiales que se deben tomar en cuenta son la verificación de la planicidad de las caras, la condición de humedad al salir de la cámara húmeda, el aseguramiento de que la máquina de ensayos cumpla con los requisitos establecidos en la norma y la adecuada toma y resguardo de los datos.

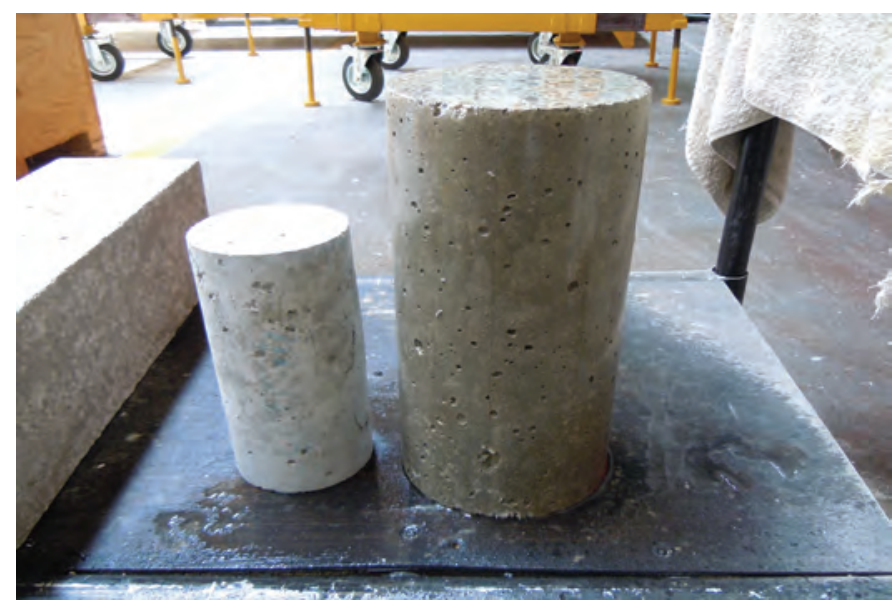

Figura 10. Espécimen húmedo para la falla. Notar la diferencia con el espécimen seco a la izquierda

\section{Referencias}

1. American Concrete Institute. ACI 318-08: Requisitos De Reglamento Para Concreto Estructural y Comentario. ACl-Capítulo Costa Rica. Costa Rica, 2008.

2. American Standard for Testing and Materials. ASTM C 123110a: Standard Practice for Use of Unbonded Caps in Determination of Compressive Strength of Hardened Concrete Cylinders. 5 páginas. Estados Unidos.

3. American Standard for Testing and Materials. ASTM C 17210: Standard Practice for Sampling Freshly Mixed Concrete. 4 páginas. Estados Unidos.

4. American Standard for Testing and Materials. ASTM C 31-10: Standard Practice for Making and Curing Concrete Test Specimens in the Field. 8 páginas. Estados Unidos.

5. American Standard for Testing and Materials. ASTM C 3910: Standard Test Method for Compressive Strength of Cylindrical Concrete Specimens. 7 páginas. Estados Unidos.

6. American Standard for Testing and Materials. ASTM C 47009: Standard Specification for Molds for Forming Concrete Test Cylinders Vertically. 4 páginas. Estados Unidos

7. American Standard for Testing and Materials. ASTM C 51109: Standard Specification for Mixing Rooms, Moist Cabinets, Moist Rooms, and Water Storage Tanks Used in the Testing of Hydraulic Cements and Concretes. 3 páginas. Estados Unidos

8. American Standard for Testing and Materials. ASTM C 61710: Standard Practice for Capping Cylindrical Concrete Specimens. 6 páginas. Estados Unidos.

9. American Standard for Testing and Materials. ASTM C 9411a: Standard Specification for Ready-Mixed Concrete. 12 páginas. Estados Unidos.

10. Neville, A. Tecnología del Concreto. Primera Edición. Instituto Mexicano del Cemento y del Concreto. México, 1999. 